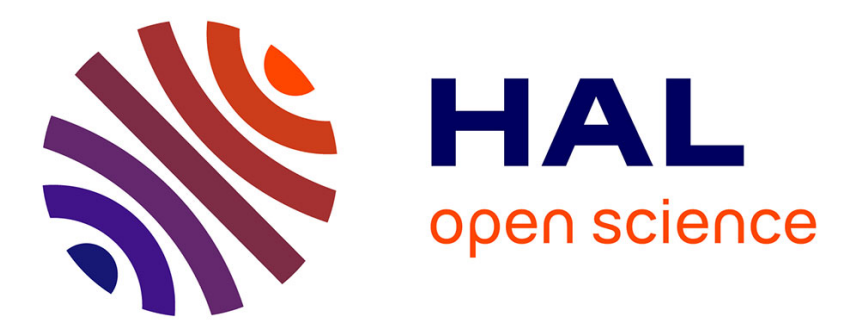

\title{
Switching the magnetic configuration of a spin valve by current-induced domain wall motion
}

J. Grollier, D Lacour, V. Cros, A. Hamzic, A. Vaurès, A. Fert, D. Adam, G. Faini

\section{- To cite this version:}

J. Grollier, D Lacour, V. Cros, A. Hamzic, A. Vaurès, et al.. Switching the magnetic configuration of a spin valve by current-induced domain wall motion. Journal of Applied Physics, 2002, 92 (8), pp.4825-4827. 10.1063/1.1507820 . hal-02949465

\section{HAL Id: hal-02949465 \\ https://hal.science/hal-02949465}

Submitted on 25 Sep 2020

HAL is a multi-disciplinary open access archive for the deposit and dissemination of scientific research documents, whether they are published or not. The documents may come from teaching and research institutions in France or abroad, or from public or private research centers.
L'archive ouverte pluridisciplinaire HAL, est destinée au dépôt et à la diffusion de documents scientifiques de niveau recherche, publiés ou non, émanant des établissements d'enseignement et de recherche français ou étrangers, des laboratoires publics ou privés. 


\title{
COMMUNICATIONS
}

\section{Switching the magnetic configuration of a spin valve by current- induced domain wall motion}

\author{
J. Grollier, D. Lacour, V. Cros, A. Hamzic, ${ }^{\text {a) }}$ A. Vaurès, and A. Fert ${ }^{\text {b) }}$ \\ Unité Mixte de Physique CNRS-THALES, Domaine de Corbeville, 91404 Orsay Cedex, \\ France and Université Paris-Sud, 91405 Orsay Cedex, France \\ D. Adam \\ THALES Research and Technology France, Domaine de Corbeville, 91404 Orsay, France \\ G. Faini \\ Laboratoire de Photonique et de Nanostructures, LPN-CNRS, Route de Nozay, 91460 Marcoussis, France
}

(Received 27 May 2002; accepted for publication 24 July 2002)

\begin{abstract}
We present experimental results on the displacement of a domain wall by injection of a dc current through the wall. The samples are $1-\mu \mathrm{m}$-wide long stripes of a $\mathrm{CoO} / \mathrm{Co} / \mathrm{Cu} / \mathrm{NiFe}$ classical spin-valve structure. The stripes have been patterned by electron-beam lithography. A neck has been defined at $1 / 3$ of the total length of the stripe and is pinning center for the domain walls, as shown by the steps of the giant magnetoresistance curves at intermediate levels $(1 / 3$ or $2 / 3)$ between the resistances corresponding to the parallel and antiparallel configurations. We show by electric transport measurements that, once a wall is trapped, it can be moved by injecting a dc current higher than a threshold current of the order of magnitude of $10^{7} \mathrm{~A} / \mathrm{cm}^{2}$. We discuss the different possible origins of this effect, i.e., local magnetic field created by the current and/or spin transfer from spin-polarized current. (C) 2002 American Institute of Physics. [DOI: 10.1063/1.1507820]
\end{abstract}

The conventional way to switch the magnetic configuration of a spin electronic device is by generating a magnetic field with an external current line. For submicronic devices, this has several drawbacks in terms of energy consumption and risk of cross talk. A recently proposed alternative way rests on passing an electrical current through the device to switch its magnetic configuration, either by spin transfer from a spin polarized current or by using the current-induced Oersted field. The magnetization reversal of a small dot by spin transfer predicted by Slonczewski ${ }^{1}$ and Berger ${ }^{2}$ has now been confirmed by experiments on multilayered pillars ${ }^{3,4}$ or nanowires, ${ }^{5}$ and magnetic switching by the current-induced Oersted field has also been observed in other types of multilayered pillars. ${ }^{6,7}$

In systems in which the magnetic configuration is defined by domains separated by domain walls (DW), a possible mechanism of magnetic switching is also the so-called current-induced domain wall drag. There are several origins of the interaction between a DW and an electrical current: the hydromagnetic drag force, which arises from the Hall effect and is not significant for very thin films, ${ }^{8}$ the currentinduced field (Oersted field) and the spin transfer by $s-d$ interaction if the current is spin polarized. This last effect, predicted theoretically by Berger, ${ }^{9}$ has an origin similar to

\footnotetext{
${ }^{a)}$ On leave from the Department of Physics, Faculty of Science, HR-10000 Zagreb, Croatia.

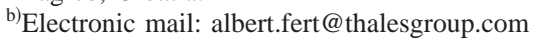

the spin transfer mechanism referred to above. It arises from the $s-d$ exchange interaction between the spin polarized electrons carrying the current and the local moments. The $s-d$ interaction exerts a torque on the spins of the conduction electrons passing through a DW and rotates the polarization direction of the current. Inversely, the spin polarized current exerts an $s-d$ exchange torque on the DW magnetic configuration and thus can give rise to a motion of the DW. The DW drag by spin transfer can be significant for thin enough DW in which the conduction electron spins cannot follow completely the local magnetization direction. This condition can be compared to the nonadiabatic criteria that has to be fulfilled in order to observe DW magnetoresistance. ${ }^{10}$ Berger et al. ${ }^{11}$ have obtained some experimental evidence of DW drag by injecting high dc current pulses in thin films and observing DW position by Kerr microscopy. The authors ascribe the DW drag to $s-d$ exchange (spin transfer). In recent experiments on 100-160 nm thick permalloy films, Gan et al. ${ }^{12}$ have also observed DW displacement due to current pulses by imaging the DW before and after the pulse using MFM. Their results suggest a combination of spin transfer and hydromagnetic DW drag. The key points in these experiments are, first that the direction of the DW displacement is reversed when the direction of dc current pulses is reversed, and second that the order of magnitude of the current pulses needed to move the DW is always $10^{7} \mathrm{~A} / \mathrm{cm}^{2}$.

The objective of the present work is to demonstrate that DW drag can be used to switch the magnetic configuration of 


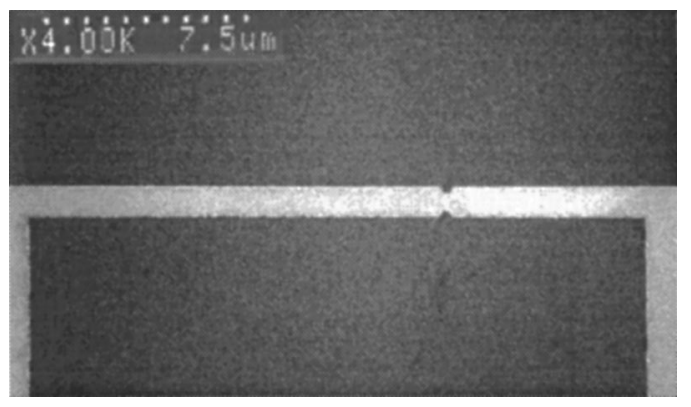

FIG. 1. SEM photography of the trilayer strip and the neck. The width of the stripe is 1 and $0.5 \mu \mathrm{m}$ in the constriction.

a magnetic device, a spin valve structure in this communication. We have used sputtering and e-beam lithography to fabricate $1 \mu \mathrm{m}$ wide and $20 \mu \mathrm{m}$ long stripes of a $\mathrm{CoO} 30 \AA / \mathrm{Co}$ $70 \AA / \mathrm{Cu} 100 \AA / \mathrm{NiFe} 100 \AA$ spin valve-type multilayered structure. A constriction ( $0.5 \mu \mathrm{m}$ wide neck) is also patterned at one third of the length, as shown in the SEM image of Fig. 1. The depth of the notches is $0.25 \mu \mathrm{m}$ and their shape is approximately triangular with a basis of about $0.3 \mu \mathrm{m}$. The antiferromagnetic $\mathrm{CoO}$ layer is used to pin the magnetization of the Co layer and to obtain well defined parallel and antiparallel configurations by reversing the magnetization of the soft permalloy layer (minor cycles). As the Néel temperature of the antiferromagnet $\mathrm{CoO}$ is under $200 \mathrm{~K}$, we have performed the experiments at low temperature $(3 \mathrm{~K})$. The stripe geometry with a neck has proved to be efficient to trap a DW at the neck and to detect its pinning and depinning directly by giant magnetoresistance (GMR) measurements. ${ }^{13}$ The small width of our stripes allows us to inject a high current density without overheating and thus to avoid the use of current pulses in contrast to Refs. 11 and 12. The resistance is measured with a standard four contact dc technique, and a magnetic field is applied along the long side of the stripe.

Figure 2 shows an example of a GMR curve (a minor cycle, with the Co moment pinned in the positive field direction) for which the measuring current was $5 \mu \mathrm{A}$ and the field resolution 1 Oe. The steps at intermediate levels $(1 / 3$ and

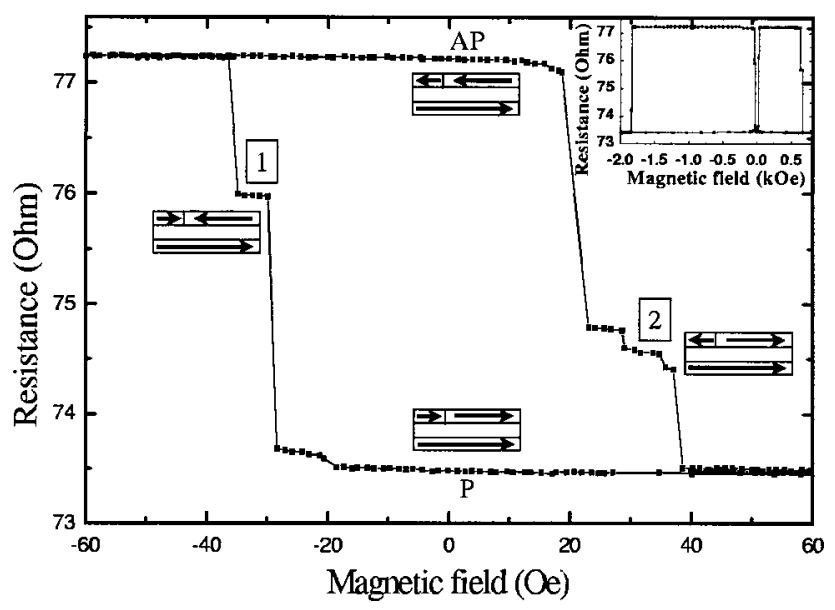

FIG. 2. Magnetoresistance curve obtained at $3 \mathrm{~K}$ with the magnetic field applied along the stripe. The loop is a minor cycle showing the permalloy reversal, whereas the complete loop is plotted in the inset.

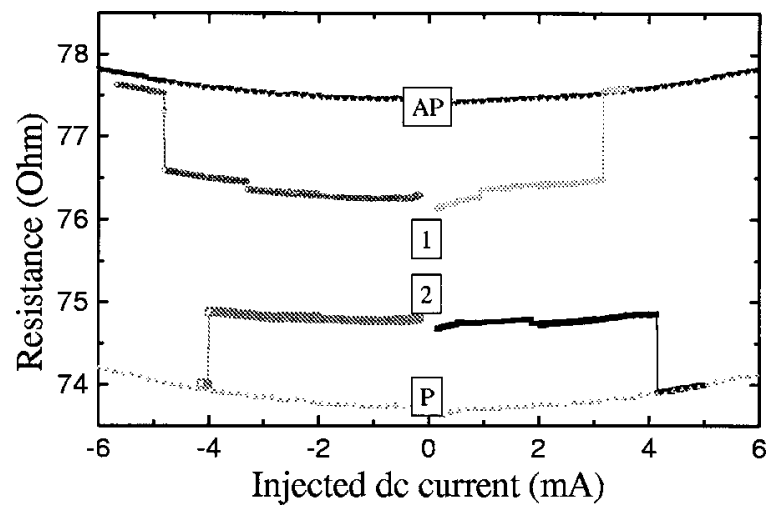

FIG. 3. Resistance vs current curves. States 1 and 2 correspond to those indicated on the GMR curve of Fig. 2.

$2 / 3$ ) between the resistances of the parallel and antiparallel configurations are clearly seen. This is the proof that the DW is trapped at the neck, as illustrated by the images in Fig. 2 .

In order to study the effect of a dc current on the DW, the following procedure was used. The field cycling is stopped at one of the intermediate steps of the minor loop. In a first set of experiments, we stop at state 1 corresponding to a -27 Oe applied magnetic field. Then, keeping the field constant, we increase or decrease the current. The variation of the resistance as a function of the current is shown in Fig. 3. The resistance first remains practically at its initial value, exhibiting only a slight reversible increase due to some heating of the sample. By comparing this resistance increase to the resistance versus temperature curve, we have estimated that the maximum increase of temperature in our experiments does not exceed $30 \mathrm{~K}$, what, as we have checked, ${ }^{14}$ is definitely insufficient to depin the DW. Then, when the current reaches a threshold value (critical current) of about 4 $\mathrm{mA}$, the resistance jumps to the level corresponding to the AP configuration, which is the more stable state in a negative field. When the experiment is repeated starting from state 2 with a +27 Oe field, at the same threshold current, the resistance jumps to the value of the stable $\mathrm{P}$ configuration.

For clarity, in Fig. 3 we added the resistance versus current curves obtained in the $\mathrm{P}$ and $\mathrm{AP}$ configurations and the vertical jumps from an intermediate resistance level to the levels of these two stable configurations are clearly observable. These jumps are the signature of DW depinning and displacement when the current exceeds a threshold value. We have also found that, once the system is in the monodomain $\mathrm{P}$ or AP configuration, it cannot be driven back to a pinned configuration (intermediate level of resistance) by varying the current.

The absolute values of the depinning critical currents in repeated experiments are scattered between 1.5 and $5 \mathrm{~mA}$. It can be pointed out that a current of $4 \mathrm{~mA}$ corresponds to a density of current crossing the neck of $2.6 \times 10^{7}$, and 5 $\times 10^{6} \mathrm{~A} / \mathrm{cm}^{2}$ if we consider only the current within the $\mathrm{NiFe}$ layer. This is in agreement with the order of magnitude given by Berger et al. and Gan et al. ${ }^{11,12}$ However a crucial point in our experiments is that the effect is symmetric with respect to the sign of the current, i.e., the DW is moved in the same direction for both current directions (cf. Fig. 3). This is 
in contradiction with the theoretical predictions ${ }^{9}$ for $\mathrm{DW}$ drag by spin transfer (and would also be in contradiction with a hydromagnetic mechanism, which in any case, is not considered for our very thin layers). This is also in contrast with the recent MFM observation of DW motion in permalloy films. ${ }^{12}$

Another possible origin of DW drag is the currentinduced (Oersted) field. The switching current of $4 \mathrm{~mA}$ should induce an in-plane transverse field of 100 Oe. This field is much larger than the coercive field of the DW, but it has no component along the stripe that could be added to the applied field and directly contribute to the depinning. The longitudinal component of the Oersted field is in average zero in the neck, but can reach local values up to a few tens of Oe, due to the neck geometry. This leads us to consider a possible twist and destabilization of the DW related to the inhomogeneity of the current-induced field. It should also be emphasized that a similar DW twist and depinning induced by the inhomogeneity of the spin transfer torque cannot be ruled out. In other words, for the specific geometry of the constriction, domain drag by spin transfer could also present different features than for DW in standard films.

In conclusion, we have shown that a dc current can switch the magnetic configuration of a spin valve structure by displacing a domain wall pinned by a constriction. The origin of the effect is not clearly established: we are not able to explain our results by the spin transfer model worked out for standard DW, or by the effect of the field generated by the current. Experiments with smaller constrictions should be useful to discriminate the two mechanisms. On the other hand, from a purely technological point of view, our finding of current-induced switching in a spin valve device indicates a promising way to control the spin electronic devices. Switching back and forth the configuration of a device by moving a domain wall between two constrictions in a nanosecond time scale should be the next step in this direction.

This work was supported by the EU through the RTN "Computational Magnoelectronics" (HPRN-CT-200000143) and the Ministère de la Recherche et de la Technologie through the MRT "Magmem II" (01V0030) and the ACI Contract "BASIC" (27-01).

${ }^{1}$ J. Slonczewski, J. Magn. Magn. Mater. 159, 1 (1996).

${ }^{2}$ L. Berger, Phys. Rev. B 54, 9353 (1996).

${ }^{3}$ J. A. Katine, F. J. Albert, R. A. Buhrman, E. B. Myers, and D. C. Ralph, Phys. Rev. Lett. 84, 3149 (2000); F. J. Albert, J. A. Katine, R. A. Buhrman, and D. C. Ralph, Appl. Phys. Lett. 77, 3809 (2000).

${ }^{4}$ J. Grollier, V. Cros, A. Hamzic, J. M. George, H. Jaffrès, A. Fert, G. Faini, J. Ben Youssef, and H. Legall, Appl. Phys. Lett. 78, 3663 (2001).

${ }^{5}$ J.-E. Wegrowe, A. Fábián, Ph. Guittienne, X. Hoffer, D. Kelly, J.-Ph. Ansermet, and E. Olive, Appl. Phys. Lett. 80, 3775 (2002).

${ }^{6}$ K. Bussmann, G. A. Prinz, S. F. Cheng, and D. Wang, Appl. Phys. Lett. 75, 2476 (1999).

${ }^{7}$ J. A. Katine, F. J. Albert, and R. A. Buhrman, Appl. Phys. Lett. 76, 354 (2000).

${ }^{8}$ W. J. Carr, J. Appl. Phys. 45, 394 (1974); L. Berger, J. Phys. Chem. Solids 35, 947 (1974).

${ }^{9}$ L. Berger, J. Appl. Phys. 55, 1954 (1984); 71, 2721 (1992).

${ }^{10}$ M. Viret, D. Vignoles, D. Cole, J. M. D. Coey, W. Allen, D. S. Danile, and J. F. Gregg, Phys. Rev. B 53, 8464 (1996).

${ }^{11}$ P. P. Freitas and L. Berger, J. Appl. Phys. 57, 1266 (1985); C.-Y. Hung and L. Berger, ibid. 63, 4276 (1988); C.-Y. Hung, L. Berger, and C. Y. Shih, ibid. 67, 5941 (1990); E. Salhi and L. Berger, ibid. 73, 6405 (1993); 76, 4787 (1994).

${ }^{12}$ L. Gan, S. H. Chung, K. H. Aschenbach, M. Dreyer, and R. D. Gomez, IEEE Trans. Magn. 36, 3047 (2000).

${ }^{13}$ T. Ono, H. Miyajima, K. Shigeto, and T. Shinjo, Appl. Phys. Lett. 72, 1116 (1998).

${ }^{14} \mathrm{DW}$ was pinned at state 1 at $3 \mathrm{~K}$ and with a $-27 \mathrm{Oe}$ magnetic field. Then, at constant field, the temperature was increased up to $150 \mathrm{~K}$ then down again to $3 \mathrm{~K}$. The resistance at the end of this temperature cycle was found to be unchanged from that obtained at state 1 at the beginning of the experiment. Thus a temperature increase much larger than the currentinduced heating does not depin the DW. 